\title{
Screening Obstructive Sleep Apnoea Syndrome from Electrocardiogram Recordings Using Support Vector Machines
}

\author{
AH Khandoker, CK Karmakar, M Palaniswami
}

The University of Melbourne, Victoria 3010, Australia

\begin{abstract}
A machine learning technique [support vector machines (SVM)] for automated recognition of obstructive sleep apnoea syndrome OSAS types from their nocturnal ECG recordings is investigated. Total 70 sets of nocturnal ECG recordings [35 sets (learning set) and 35 sets (test set)] from normal subjects (OSAS-) and subjects with OSAS (OSAS+) were collected from physionet. Features extracted from successive wavelet coefficient levels after wavelet decomposition of $R R$ intervals and QRS amplitudes of whole record were presented as inputs to train the SVM mode to recognize OSAS+/- subjects. The optimally trained SVM showed that a SVM using a subset of selected combination of $H R V$ and EDR features correctly recognized 20 out of 20 OSAS+ subjects and 10 out of 10 OSAS- subjects. For estimating the relative severity of OSAS, the posterior probabilities of SVM outputs were calculated.
\end{abstract}

\section{Introduction}

Obstructive sleep apnoea syndrome (OSAS), commonly known as sleep apnea is defined by frequent cessation of breathing due to the partial or complete obstruction of upper airway for short periods during sleep. The fragmented sleep due to OSAS can result in poorer daytime cognitive performance, increased risk of motor vehicle and workplace accidents, depression, diminished sexual function, and memory loss [1]. Undiagnosed OSAS is now regarded as an important risk factor for the development of cardiovascular diseases (e.g. hypertension, stroke, congestive heart failure, left ventricular hypertrophy, acute coronary syndromes) [2]. Therefore, early recognition of subjects at risk of OSAS is essential.

Based on early in the investigation, it was recognised that the events of apnoea are accompanied by concomitant cyclic variations in R-R intervals (beat to beat heart rate) of ECG signals [3]. A comparative study [4] on different algorithms for apnoea detection based on
ECG signals reported that the combination of parameters of HRV and the EDR signal gave the best OSAS recognition results. Parameters related to cardiac and respiratory dynamics can readily be extracted from heart rate variability (HRV) and ECG derived respiration (EDR) signals [5]. However, after deriving and combining suitable index parameters, an appropriate computational method must be chosen to discriminate between healthy and OSAS subjects. Several machine learning techniques, i.e., Linear and Quadratic Discrimant model [6], CART method [7] have been used for automatic recognition of OSAS subjects based on the selected subset of parameters derived from HRV and EDR signals.

Recently, support vector machines (SVM) have emerged as a powerful technique for general purpose pattern recognition [8-9]. The primary advantage of SVM is its ability to minimize both structural and empirical risk [10] leading to better generalization for new data classification even with limited training data set. Therefore, it was hypothesized that an SVM model would be suitable for constructing relationship between features extracted from ECG signals and the presence or absence of OSAS. Thus, in this study, we propose to apply SVM for automated recognition of OSAS based on overnight ECG signals.

\section{Methods}

Overnight sleep ECG signals were collected from Physionet Apnea-ECG database (www.physionet.org). The database was divided into two sets each containing 35 recordings. The first set (learning) was used to optimize the classification algorithm and the second set (test) was used to provide an independent performance assessment.

Amplitudes of each QRS complex [5] and the intervals between successive $\mathrm{R}$ waves of QRS complex were determined with $1 \mathrm{~ms}$ accuracy of precision for all recordings using an algorithm described in another study [11]. Each RR-intervals series was divided in subintervals 
of 5 points each. Through a moving average procedure we deleted suspect RR interval if its value exceeds by more than $20 \%$ of the median value of RR intervals during each subinterval. Then, HRV and EDR signals were resampled using cubic spline interpolation to make 32768 points for wavelet (Wv) decomposition at 14 levels using daubechies wavelets with order 10 and feature extraction. In order to eliminate the bias of mean and variance of the signal on feature extraction, all signals were normalized by calculating their $z$-scores (i.e., $(\mathrm{x}-\mu) / \sigma$, where $\mu$ is the mean and $\sigma$ is the standard deviation for the feature). Then features were used as input features to the SVM model, and an output representing the OSAS types $(-1=$ OSAS-, $+1=$ OSAS +$)$ for learning the complex relationship of the HRV and EDR patterns with the potential of OSAS. All SVM architectures were trained and tested on the MATLAB SVM toolbox [10]. SVM is an approximate implementation of the method of "structural risk minimization" aiming to attain low probability of generalization error and finds the optimal separating hyperplane $(\mathrm{OSH})$ by maximizing the margin between the classes [8]. Regularization parameter ('C') determines the trade off between the maximum margin and minimum classification error. SVM first transforms input data into a higher dimensional space by means of a kernel function and then constructs a linear (OSH) between the two classes in the transformed space. Those data vectors nearest to the constructed line in the transformed space are called the support vectors (SV). An overview of SVM pattern recognition can be found in $[8,10]$.

In this study, we experimented with three kernels as follows. 1) Linear kernel, $K\left(\mathbf{x}_{i}, \mathbf{x}_{j}\right)=\mathbf{x}_{i} \cdot \mathbf{x}_{j}$

2)Polynomial kernel, $K\left(\mathbf{x}_{i}, \mathbf{x}_{j}\right)=\left(\mathbf{x}_{i} \cdot \mathbf{x}_{j}+1\right)^{d} d$ is the degree of polynomial

3) Radial basis function(RBF) kernel, $K\left(\mathbf{x}_{i}, \mathbf{x}_{j}\right)=\exp \left(-\frac{\left\|\mathbf{x}_{i}-\mathbf{x}_{j}\right\|^{2}}{2 \sigma^{2}}\right) \sigma$ is the width of RBF

\section{Results and discussion}

Although enhancement of the coefficients for the levels 256 to 2 in the OSAS+ subject (Fig 1) can be visually noted, compared with the OSAS- subject, variances of the coefficients at levels 2048, 512, 256, 32 and 16 are significantly different (student $t$ test; $p<0.001$; Table 1) between the two groups. On the other hand, variance of the coefficient level 2 of EDR signals (Fig. 1) was found to be significantly different between two
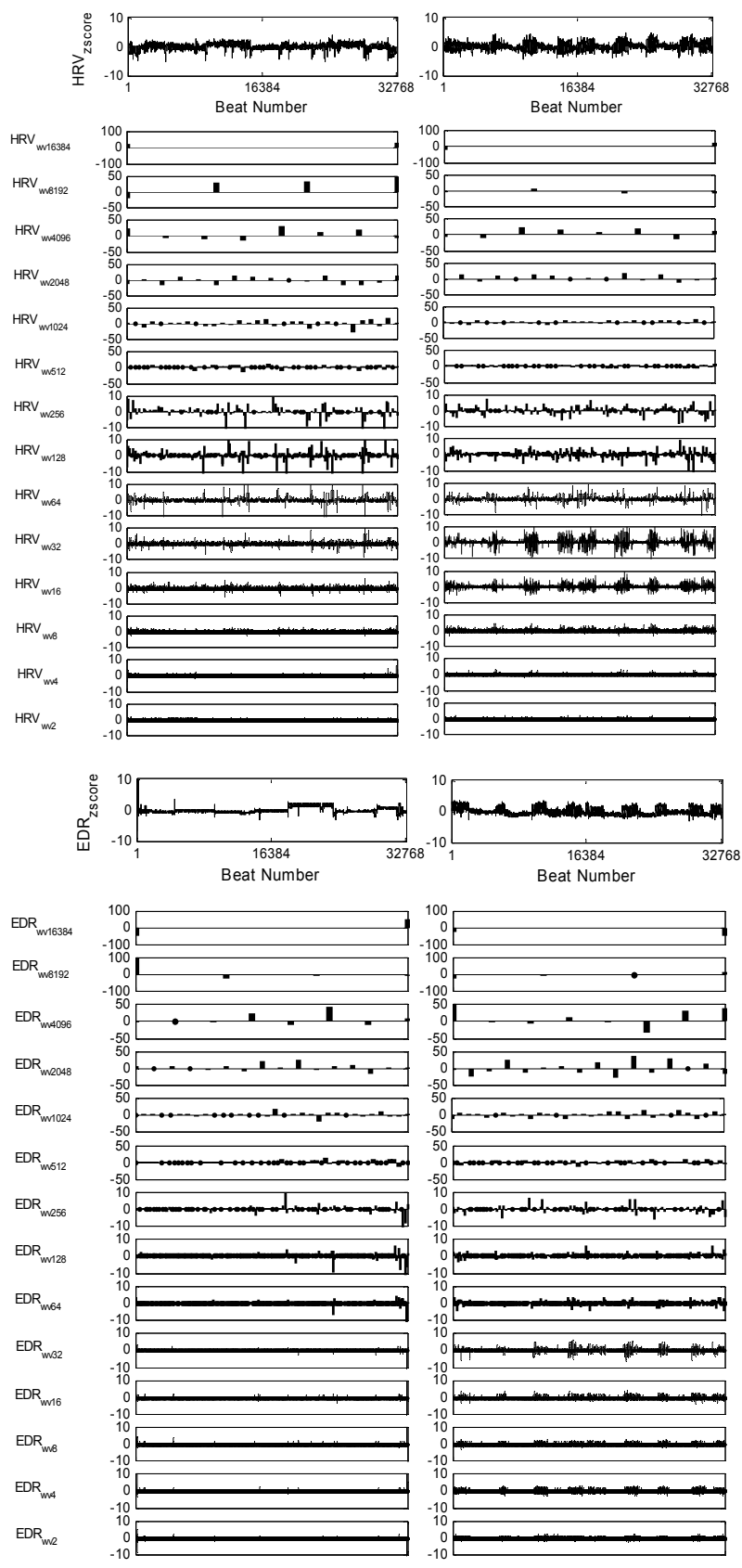

(A)

(B)

Figure 1. Wavelet decomposition of heart rate variability $(\mathrm{HRV})$ and ECG derived respiration (EDR) signals into fourteen levels for A) an OSAS- (file c02; apnea period 1 min; $\mathrm{AHI}=0.12$ ) subject and $\mathrm{B}$ ) an OSAS+ (file a19; apnea period $204 \mathrm{~min}$; $\mathrm{AHI}=24.43$ ) subject during 32768 samples

groups ( $\mathrm{p}<0.001$, Table 1). Since the changes in EDR are due to the movement of the chest electrodes during 
respiration, the sleeping position of the subject have influenced on the EDR signals (Fig. 1 bottom panel).

In order to provide the relative importance of features, receiver-operating curve (ROC) analysis was used. ROC curves were built separately for each feature and areas under all ROC curves were calculated and summarized in Table 1. The single best HRV feature was found to be HRVwv32 (ROCarea $=0.99, p<0.01$ ) and the best EDR feature was EDRWv2 (ROCarea $=0.77, \quad \mathrm{p}<0.01)$. HRVwv32 corresponds to LF component $(0.0625 \mathrm{~Hz})$ indicating apnea specific cyclic oscillation pattern of heart rate rhythm. EDRwv2 corresponds to HF components caused by respiration.

Considering $14 \mathrm{HRV}, 14 \mathrm{EDR}$ and 28 combined (HRV+EDR) features as input of SVM, classification performance was separately tested in ROC curves built for polynomial $(\mathrm{d}=3 ; \mathrm{C}=1)$ kernel. Combined (HRV+EDR) features showed overall better performance (ROCarea $=0.90$ ) compared to individual feature types (ROCarea $=0.87$ for HRV and 0.50 for EDR features).

A leave-one-out cross-validation scheme was adopted to evaluate the generalization ability of the classifier. Using Polynomial $(\mathrm{d}=3 ; \mathrm{C}=1)$, RBF $(\sigma=1.0 ; ; \mathrm{C}=1)$ and linear kernels classification accuracy plotted as a function of features selected by (hill-climbing) feature selection algorithm [9] was presented in Fig. 2. The first best subset of all features selected by the algorithm to achieve maximum accuracy $(96.67 \%)$ was found to be $\{10,28,12,9,20\}$ using polynomial kernel. Performance remained unchanged for adding features 13,14 and 26. When linear kernel was used, the first best subset was found to be $\{10,5,9,21\}$ and addition of feature 12,28 and $13 \mathrm{did}$ not alter the classification performance (accuracy $=96.67 \%$ ). Overall, it emphasizes that classifier was able to discriminate well when trained with a subset comprising a few good features. Compared to performances of RBF kernel, linear and polynomial kernel performed better on learning set. Kernel parameter (d) was varied from 1 to 20 to generate models on the basis of the highest accuracy on the validation set is taken. Overall performance of the classifier was found to be highest $(96.67 \%)$ at $\mathrm{d}=3$.

Results of independent test on classification performance (overall accuracy, sensitivity and specificity) of the SVM classifier as a function of number of HRV and EDR features showed that 100\% accuracy was obtained using polynomial kernel $(\mathrm{d}=3$ and $\mathrm{C}=0.1,1,10)$ with four subsets of features $[\{10,28,12,9$, $20\},\{10,28,12,9,20,13\},\{10,28,12,9,20,13$, $14\},\{10,28,12,9,20,13,14,26\}]$. However, linear kernel performed the same accuracy when only two subsets of features with $C=10.0[\{10,5,9,21,12,28\}$, $\{10,5,9,21,12,28,13\}]$ were used.

Besides the recognition of OSAS types (+/-), it was interesting to look at the influence of observations from the available samples on the probability of being OSAS+. In order to estimate the relative severity of apnoea for each individual of the test sets ( 35 records), the posterior probabilities of SVM classifier outputs were calculated using the method described by Platt [12]. The posterior probabilities (shown in Fig. 3) can provide an estimate of the severity of apnoea ( 0 indicates no apnoea whereas a value close to 1 indicates severely apnoea).Within 35 subjects' sample, threshold probability level was set at 0.5 . Out of 5 borderline subjects, 3 subjects were in the range of probability $0.50 \sim 0.55$. However, 2 subjects were found to be equal or above the 0.70 probability.

Table 1. $p$ value and area under ROC curves of features extracted from wavelet analyses of HRV signals and EDR signals. ${ }^{*}$ indicates the significance at $p<0.01$.

\begin{tabular}{clcc}
\hline $\begin{array}{c}\text { Feature } \\
\text { No. }\end{array}$ & Feature & $\boldsymbol{p}$ value & ROC Area \\
\hline 1 & $\mathrm{HRV}_{\mathrm{wv} 16384}$ & 0.3620 & 0.62 \\
2 & $\mathrm{HRV}_{\mathrm{wv} 8192}$ & 0.6779 & 0.64 \\
3 & $\mathrm{HRV}_{\mathrm{wv} 4096}$ & 0.0617 & 0.77 \\
4 & $\mathrm{HRV}_{\mathrm{wv} 2048}$ & $0.0003^{*}$ & 0.75 \\
5 & $\mathrm{HRV}_{\mathrm{wv} 1024}$ & 0.0126 & 0.79 \\
6 & $\mathrm{HRV}_{\mathrm{wv} 512}$ & $0.0027^{*}$ & 0.90 \\
7 & $\mathrm{HRV}_{\mathrm{wv} 256}$ & $0.0026^{*}$ & 0.89 \\
8 & $\mathrm{HRV}_{\mathrm{wv} 128}$ & 0.0914 & 0.81 \\
9 & $\mathrm{HRV}_{\mathrm{wv} 64}$ & 0.3340 & 0.53 \\
10 & $\mathrm{HRV}_{\mathrm{wv} 32}$ & $0.0013^{*}$ & 0.99 \\
11 & $\mathrm{HRV}_{\mathrm{wv16}}$ & $0.0006^{*}$ & 0.94 \\
12 & $\mathrm{HRV}_{\mathrm{wv8}}$ & 0.1970 & 0.76 \\
13 & $\mathrm{HRV}_{\mathrm{wv} 4}$ & 0.1250 & 0.81 \\
14 & $\mathrm{HRV}_{\mathrm{wv} 2}$ & 0.1623 & 0.51 \\
\hline 15 & $\mathrm{EDR}_{\mathrm{wv} 16384}$ & 0.2775 & 0.54 \\
16 & $\mathrm{EDR}_{\mathrm{wv} 8192}$ & 0.2174 & 0.68 \\
17 & $\mathrm{EDR}_{\mathrm{wv} 4096}$ & 0.4267 & 0.51 \\
18 & $\mathrm{EDR}_{\mathrm{wv} 2048}$ & 0.3257 & 0.64 \\
19 & $\mathrm{EDR}_{\mathrm{wv} 1024}$ & 0.7145 & 0.69 \\
20 & $\mathrm{EDR}_{\mathrm{wv} 512}$ & 0.7795 & 0.58 \\
21 & $\mathrm{EDR}_{\mathrm{wv} 256}$ & 0.9118 & 0.62 \\
22 & $\mathrm{EDR}_{\mathrm{wv} 128}$ & 0.6646 & 0.51 \\
23 & $\mathrm{EDR}_{\mathrm{wv} 64}$ & 0.4502 & 0.56 \\
24 & $\mathrm{EDR}_{\mathrm{wv} 32}$ & 0.0436 & 0.66 \\
25 & $\mathrm{EDR}_{\mathrm{wv} 16}$ & 0.2076 & 0.60 \\
26 & $\mathrm{EDR}_{\mathrm{wv} 8}$ & 0.1005 & 0.67 \\
27 & $\mathrm{EDR}_{\mathrm{wv} 4}$ & 0.0727 & 0.68 \\
28 & $\mathrm{EDR}_{\mathrm{wv} 2}$ & $0.0049^{*}$ & 0.77 \\
\hline & & & \\
\end{tabular}




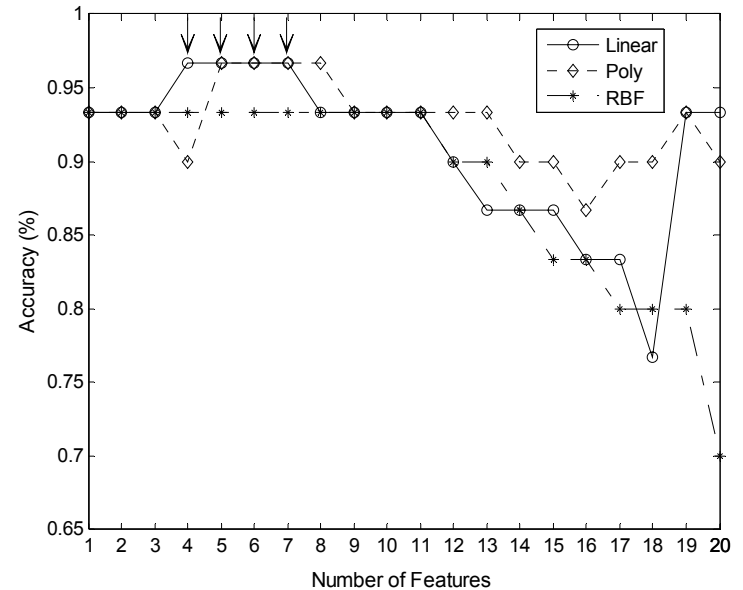

Figure 2. Dependence of \% classification accuracy for learning set on the number of features selected by "hillclimbing" feature selection Best classification performance (shown by arrow) was obtained using selected subsets of HRV +EDR features.

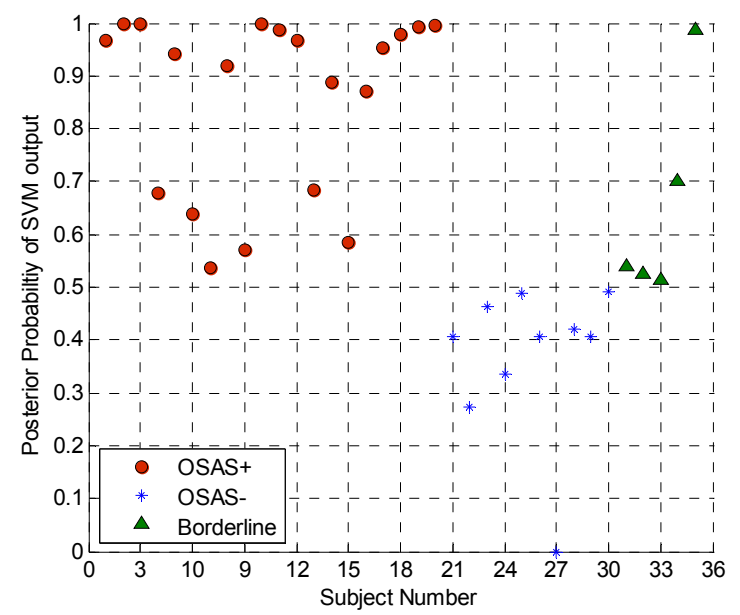

Figure 3. Posterior probability estimate $\mathrm{P}(\mathrm{y}=\mathrm{OSAS}+\mid \mathrm{f}(\mathrm{x}))$, for test subjects with OSAS $+(1-20)$, OSAS- (21-30) and borderline subjects (31-35), calculated from SVM output values for each class.

\section{Conclusions}

These results suggest superior performance of SVM in OSAS recognition based on HRV and EDR features of ECG, and demonstrate considerable potential in applying SVM in ECG based screening device that can aid sleep specialist in the initial assessment of patients with suspected OSAS.

\section{Acknowledgements}

This study was supported by an Australian Research Council (ARC) linkage project with Compumedics Pty Ltd (LP0454378).

\section{References}

[1] Coleman J. Complications of snoring, upper airway resistance syndrome, and obstructive sleep apnea syndrome in adults. Otolaryngol Clin North Am ;1999;32: 223-234.

[2] Young T, Peprad P, Palta M, Hla KM, Finn L, Morgan B. Population-based study of sleep-disordered breathing as a risk factor for hypertension. Arch. Intern. Med., 1997; 157: 1746-1752.

[3] Guilleminault C, Connolly SJ, Winkle R, Melvin K, and Tilkian A. Cyclical variation of the heart rate in sleep apnoea syndrome. Mechanisms and usefulness of $24 \mathrm{~h}$ electrocardiography as a screening technique. The Lancet, 1984; I: 126-131.

[4] Penzel T, McNames J, de Chazal P, Raymond B, Murray A, Moody G. Systematic comparison of different algorithms for apnoea detection based on electrocardiogram recordings. Med Biol Eng Comput, 2002;40:402-407.

[5] Moody GB, Mark RG, Zoccola A, Mantero S. Clinical validation of the ECG-derived respiration (EDR) technique. In: Computers in Cardiology, Piscataway NJ: IEEE Computer Society Press; 1986; 5:07-10

[6] De Chazal P, Heneghan C, Sheridan E, Reilly R, Nolan P and O'Malley M. Automated Processing of the Single Lead Electrocardiogram for the Detection of Obstructive Sleep Apnoea, IEEE Trans. Biomed. Eng, 2003; 50(6): 686 $-696$.

[7] Roche F, Pichot V, Sforza E. et al. Predicting sleep apnoea syndrome from heart period: a time-frequency wavelet analysis. Eur Respir J, 2003;22:937-942.

[8] Vapnik VN. The Nature of Statistical Learning Theory. Springer, New York, 1995.

[9] Khandoker AH, Lai D, Begg RK and Palaniswami M. Wavelet-based feature extraction for support vector machines for screening balance impairments in the elderly. IEEE Trans on Neural Systems and Rehabilitation Engg. (in press)

[10] Gunn S. Support vector machines for classification and regression. ISIS Technical Report, University of Southampton,UK. 1998.

[11] Pan J and Tompkins WJ. Real time QRS detector algorithm. IEEE Trans. Biomed. Eng., 1985; 32:230-323.

[12] Platt JC. Probabilistic outputs for support vector machines and comparison to regularized likelihood methods. In: A. Smola, P. Bartlett, B. Scholkopf, and D. Schuurmans (eds.): Advances in Large Margin Classifiers. Cambridge, MA.2001.

Address for correspondence

Ahsan Khandoker

Department of Electrical \& Electronic Engineering, The University of Melbourne, Parkville, VIC3010. Australia. a.khandoker@ee.unimelb.edu.au 